\title{
VISUALIZATION OF MAGNETICALLY CONFINED PLASMAS
}

\author{
J.L.V. Lewandowski * \\ Princeton University, \\ Princeton Plasma Physics Laboratory \\ Princeton NJ 08543, USA
}

December 3, 1999

\begin{abstract}
With the rapid developments in experimental and theoretical fusion energy research towards more geometric details, visualization plays an increasingly important role. In this paper we will give an overview of how visualization can be used to compare and contrast some different configurations for future fusion reactors. Specifically we will focus on the stellarator and tokamak concepts. In order to gain understanding of the underlying fundamental differences and similarities these two competing concepts are compared and contrasted by visualizing some key attributes.
\end{abstract}

Keywords: Fusion energy, plasma, tokamak, stellarator, stability, equilibrium, transport.

*Part of this work has been carried out at the Australian National University, Department of Theoretical Physics, Canberra ACT 0200, Australia 


\section{Introduction}

Modern civilizations are dependent on energy. In the industrialized part of the world the demand for electrical power is increasing steeply, with the consumption doubling approximately every ten years. It is hard to imagine this trend continuing but there is still a strong need to develop an environmental attractive, commercially viable, and sustainable energy source for the next century. To develop a system capable of providing such a source of energy is the mission of the fusion energy research community.

There is presently a great imbalance in the world energy consumption. As an example, in 1990, the per capita consumption of energy in India and China were $1 / 6$ and $1 / 3$ of the world average respectively. Over the next decades it is likely that these figures will increase considerably. A large part of this increase will no doubt be generated by conventional burning of fossil fuels. This will degrade the local environments greatly and the impact on the global environment is simply staggering. Hence, there is a great need to develop viable renewable energy sources and increase the efforts on developing new technologies such as those of controlled thermo-nuclear fusion.

Fusion technologies hold a great promise for the future. Widespread introduction of fusion energy power plants could substantially reduce the environmental impacts of increasing worldwide demands for electricity.

For more than fifty years, researchers from around the world have worked towards achieving controlled thermo-nuclear fusion [1,23]. Fusion is the process by which lights elements are fused together forming heavier elements and liberating energy in the process. There are two different paths to achieve this goal. In inertial fusion a small pellet of solid fuel is bombarded by photons generated by high energy lasers or ions generated by particle accelerators. The second and perhaps more promising avenue is by means of magnetic confinement. In this scheme a fully ionized gas, a so called plasma, is confined in a strong magnetic field. The goal is to confine a plasma of sufficiently high temperature and density and for a sufficiently long period of time as to allow the particles to fuse. The obvious aim is to get more energy out than what is put in. The easiest reaction to achieve thermo-nuclear fusion brings together the nuclei of two heavy isotopes of hydrogen: deuterium and tritium. The Coulomb repulsion between the nuclei are overcome by heat 
and the system are generally externally heated with quite elaborate heating systems ranging from radio waves to injection of energetic neutral particles. The following discussing will be limited to magnetically confined plasmas.

The magnetic confinement scheme relies on the fact that the presence of magnetic fields can affect the motion of the individual charged particles in the plasma. Charged particles can move relatively free along the lines of the magnetic field and the most successful devices for confining the plasma are therefore closed into a toroidal shape. Over the decades a large number of concepts of toroidal confinement have been investigated both theoretically and experimentally. Current research efforts are mainly focused on two different concepts, the tokamak [26] and the stellarator [25, 2].

The tokamak [26] produces its magnetic field both from external coils and by strong currents in the plasma generated by letting the plasma play the role of the secondary winding of a transformer. The tokamak concept is the one most favored in the world today. This is partly for historical reasons and partly because of its relatively simple geometry. However, the tokamak concept suffers from some intrinsic problems. Due to the dependence of an external transformer the tokamak is intrinsically a pulsed device. This issue is presently a primary focus of the tokamak research community and several different ways to remedy this problem are considered. Furthermore, the large currents driven by the transformer can also drive violent instabilities, the so-called disruptions. During a large disruption the whole plasma is lost and a large amount of energy is released with potential detrimental effect on experimental hardware.

Stellarators [25, 2] on the other hand are intrinsically steady state devices and are generally believed to be disruption free. In the stellarator the confining magnetic field is essentially produced by currents flowing in coils surrounding the plasma. This is obviously a great advantage when it comes to controlling the plasma but the drawback of the stellarator concept is its geometry. While the doughnut-shaped tokamak has continuous symmetry and is essentially a 2-dimensional configuration, the stellarator is fully 3-dimensional. Over the last decades there has been an resurge of interest in stellarator concept. This evolution can be attributed to the evolution of large scale supercomputers and advanced visualization making an optimization of fully 3-dimensional configurations viable. 
Visualization is about gaining understanding and insight. In the following we will show some examples of how visualization can be used in this way to compare and contrast different magnetic configurations. As examples we will particularly focus on the stellarator and tokamak concepts. The aim is to compare contrast the two concepts by visualizing key equilibrium quantities and by identifying the underlying fundamental differences and similarities. Visualization is about gaining understanding and insight. In the following we will show some examples of how visualization can be used in this way to compare and contrast different magnetic configurations. As examples we will particularly focus on the stellarator and tokamak concepts. The aim is to compare contrast the two concepts by visualizing key equilibrium quantities. Hence gaining an understanding for the underlying fundamental differences and similarities.

\section{The physics}

The plasma physics problems encountered in controlled thermo-nuclear fusion can be separated into four basic areas:

\section{- Equilibria}

The problem of equilibria deals with balancing the forces of the kinetic pressure with those of the magnetic field. Kinetic pressure attempts to move plasma away minimizing the potential energy of the system while the magnetic field tries to keep the plasma in place.

\section{- Stability}

A plasma in equilibrium can also be moved away from a given configuration by means of various instabilities. These instabilities occur in many forms and shapes and on many different time and length scales.

\section{- Transport}

Transport of energy and particles out from the hot plasma center lowers the temperature (or average kinetic energy) and particle densities and therefore deteriorates the conditions for thermo-nuclear fusion.

\section{- Heating}

Extra heating applied from the outside of the plasma is important to get to temperature levels necessary for fusion. 
The division above is somewhat artificial as the issues are directly related. The measured energy transport in magnetically confined plasmas is generally much larger than the predictions of simple estimates based on particle orbits and is often called anomalous for this reason. The discrepancy is attributed to small scale plasma turbulence. The turbulence is a result of the nonlinear evolution of small scale instabilities. The stability of the latter are sensitive to the equilibrium. The equilibrium in turn depends on the transport of energy. Thus, the issues are strictly inseparable.

To treat such a complicated coupled system self-consistently is however not tractable even with the largest of today's supercomputers. The problems are therefore decoupled with the help of further assumptions. The equilibria are solved for assuming the pressure and current profiles. In turn, the stability of these equilibria is calculated assuming the equilibria to be fixed in time. The associated turbulence is then either estimated or calculated from limited nonlinear models, again assuming the equilibria to be constant in time. Development of more complete nonlinear calculations are presently under way but has yet some way to go before a self-consistent model coupling equilibrium, stability and nonlinear simulations to predict transport in real configurations.

A large part of the annual budget of the international fusion research programme goes into the development of numerical codes to simulate real experiments. A large code is needed to treat each of the many aspects of the plasma because of the different length and time scales involved. Furthermore, the relative strength of the interactions between the issues of (dynamic or static) equilibrium, stability and turbulence-driven transport varies from place to place in the plasma. The relevant physics to be analyzed numerically or analytically also depends on the location in the plasma; for example, the outer part of the plasma (also called the edge plasma) is cold and contains significant numbers of neutral atoms and molecules: the atomic processes, such as charge exchange, recombination, radiation and interspecies collisions, have to be taken into account when modelling the edge plasma (atomic processes are much less important in the core (hot and dense) plasma). Here we briefly review the plasma models that are most widely employed in the modelling of tokamak and stellarator plasmas. The plasma models can be divided into, somewhat arbitrarily, three categories: (a) particle model; (b) kinetic model; and (c) fluid model. The particle model has its starting point 
with the equation of motion of the particle species (electrons, singly-charged ions and impurities)

$$
m_{j} \frac{d^{2} \mathbf{r}}{d t^{2}}=q_{j}\left(\mathbf{E}+\frac{\mathbf{v} \times \mathbf{B}}{c}\right) .
$$

Here $\mathbf{r}$ is the position vector of particle species $j$ with mass $m_{j}$ and charge $q_{j}$. The electric field, E, and the magnetic field, B, must be calculated selfconsistently using Maxwell's equations. The timescale associated with the equation of motion (1) is (for electrons) $\tau_{\mathrm{p}} \sim 1 / \omega_{\text {ce }}$ where $\omega_{\text {ce }} \equiv(e B) /\left(m_{\mathrm{e}} c\right)$ is the so-called electron cyclotron frequency. For typical tokamak plasma parameters, $\tau_{\mathrm{p}}$ is of the order of $10^{-11} \mathrm{~s}$. It is evident that the above model is impractical since the lifetime of a typical discharge is $\tau_{\mathrm{d}} \sim 1-10 \mathrm{~s}$, so that $\tau_{\mathrm{d}} / \tau_{\mathrm{p}} \sim 10^{11}-10^{12}$. However, in a strong magnetic field, particles move rapidly along helices of radius $\rho_{j}$; one can average over the (fast) gyro-motion and study the resulting guiding centre dynamics with relevant timescale $10^{-4} \mathrm{~s}$. In the late 70's, Okuda and co-workers have developed simple particle codes; the ion motion is governed by the Lorentz force law represented using the standard leapfrog scheme. The electrons are treated using a drift approximation, necessitating the use of a predictor-corrector scheme to treat their motion perpendicular to the field lines. Poisson's equation (which ensures the plasma quasineutrality) for the electrostatic potential is solved by Fourier transforming. The main difficulty with the codes described in Refs [28, 29, 30] is particle noise. In order to reduce particle noise, Lee [31, 32] developed a gyro-kinetic model. In the gyro-kinetic model, the set of equations (1) are averaged over the gyromotion while retaining effects due to the finite radius of the gyro-orbits. The calculation of the ion density and the method of solution of Poisson's equation are the main differences with ordinary particle codes: the ion density is calculated by introducing the concept of a ring of charge $[31,32]$; Poisson's equation is solved in wavenumber space rather than real space. To bypass the difficulty associated with the short electron timescale, the subcycling technique [33] is used for electrons. For toroidal geometries, the toroidal particle code (TPC) of LeBrun and Tajima [39] uses a non-uniform mesh for the radial coordinate. The ion are pushed in a local Cartesian system using a standard leapfrog scheme, then transformaing back to the toroidal system at every timestep. To treat a large number particles (typically $\sim 10^{20}$ ), it is convenient to introduce a number density function $F_{j}(\mathbf{r}, \mathbf{v}, t)$ such that $F_{j} d^{3} r d^{3} v$ represents the number of particles of species $j$ in the phase space elementary volume $d^{3} r d^{3} v$. The distribution function $F_{j}$ 
is governed by Boltzmann's equation

$$
\frac{d F_{j}}{d t} \equiv \frac{\partial F_{j}}{\partial t}+\mathbf{v} \cdot \nabla F_{j}+\frac{q_{j}}{m_{j}}\left(\mathbf{E}+\frac{\mathbf{v} \times \mathbf{B}}{c}\right) \cdot \nabla_{\mathbf{v}} F_{j}=\sum_{k \neq j} \mathcal{C}_{j k}\left(F_{j}, F_{k}\right)
$$

where $\boldsymbol{\nabla}_{\mathbf{v}}$ is the gradient operator in velocity space and $\mathcal{C}_{j k}\left(F_{k}, F_{j}\right)$ is the collision operator between species $j$ and $k$. In a hot plasma, one may take, in first approximation, $\mathcal{C}_{j k} \approx 0$ and equation (2) reduces to the so-called Vlasov equation. Writting $F=F_{0}+\delta F$, where $F_{0}$ is the equilibrium (Maxwellian) distribution function, one can obtain an evolution equation for $\delta F$

$$
\left(\frac{\partial}{\partial t}+\mathbf{v} \cdot \nabla_{6}\right) \delta F=S_{0}\left(F_{0}\right) \equiv S_{0}
$$

Equation (3) can be regarded as an evolution equation for $\delta F$ with a source $S_{0}$. As discussed by Tajima [38], each particle is assigned a weight $w_{j}$ such that

$$
\delta F=\sum_{j} w_{j} \delta\left(\mathbf{r}-\mathbf{r}_{j}(t)\right) .
$$

Alternatively, one may write $\delta F=W F_{\star}$, where $W$ is a weighting function, and $F_{\star}$ satisfies

$$
\dot{W} F_{\star}+W \dot{F}_{\star}=S_{0},
$$

where an overdot denotes the derivative along a trajectory. There is some freedom in specifying the weighting function $W$; for some specific cases, the reader can consult, for example, Ref [38]. Standard fluid models are obtained by taking moments

$$
M_{k} \equiv \int \mathbf{v}^{k} \mathcal{L}[F] d^{3} v
$$

of Boltzmann's equation. Here $k=0,1,2, \ldots$ and $\mathcal{L}$ denotes the global operator given in Boltzmann's equation (2)

$$
\mathcal{L} \equiv \frac{\partial}{\partial t}+\mathbf{v} \cdot \boldsymbol{\nabla}+\frac{q_{j}}{m_{j}}\left(\mathbf{E}+\frac{\mathbf{v} \times \mathbf{B}}{c}\right) \cdot \nabla_{\mathbf{v}}-\mathcal{C} .
$$

For many applications, velocity space effects can be neglected so that only the first few moments (6) of the velocity distribution $F_{j}$ can be retained. It is easy to show that the equation for $Q_{k} \equiv \int \mathbf{v}^{k} F_{j} d^{3} v$ involves, in general, all the moments up to $Q_{k+1}$ (inclusive). Therefore one obtains an infinite set of 
equations and one must truncate the complete set by introducing an appropriate closure method. If the distribution function $F_{j}$ does not depart strongly from a Maxwellian distribution (that is $F_{j} \approx F_{\mathrm{M} j}=A \exp \left[-m v^{2} /\left(2 k_{\mathrm{B}} T_{j}\right)\right]$ where $A$ is a constant of normalization), one can obtain approximate expressions for the various (classical) transport coefficients; see for instance the monograph of Braginskii [3]. The set of Braginskii's fluid equations [3] offers a relatively simple starting point to the problem of slow (frequency much smaller than the ion cyclotron frequency), drift-type $\left(k_{\|} / k_{\perp} \ll 1\right.$ where $k_{\|}$ and $k_{\perp}$ are the parallel and perpendicular components of the wavevector, respectively) modes; these modes, also referred to as drift waves, are believed to be responsible to the large (anomalous) transport accross the magnetic field lines observed in tokamak [4] and stellarator [5] plasmas. We do not discuss the derivation of the various fluid models for drift waves that can be found in the literature, e.g. Refs $[7,8]$, but consider a simple model for illustrative purposes. From the ion momentum and continuity equations, it is possible to derive a prototypical one-field model for drift waves; neglecting the ion motion along the field line, one can show the fluctuating electrostatic potential $\Phi$ is governed by the following equation (in appropriate normalized units) [9]

$$
\left(\frac{\partial}{\partial t}+\mathbf{v}_{E} \cdot \boldsymbol{\nabla}\right)\left[N(\Phi)-\nabla^{2} \Phi\right]=0
$$

where $\mathbf{v}_{E} \equiv c(\mathbf{E} \times \mathbf{B}) / B^{2}$ is the (lowest-order) cross-field drift velocity of the ions. Here $N$ is the total plasma density and $\mathbf{E} \simeq-\nabla \Phi$ is the fluctuating electric field. Equation (8) was introduced by Terry and Horton and it is sometimes called the Terry-Horton equation [9]. Various models for the plasma density $N$ can be studied; for instance, if $N_{0}$ represents the equilibrium plasma density, than one possible model is

$$
N(\Phi)=N_{0}+(1-i \delta) \Phi
$$

where $\delta \geq 0$; in the special case $\delta=0$, the perturbed plasma density is adiabatic, the drift wave is marginally stable and the Terry-Horton equation reduces to the Hasegawa-Mima equation [6]. Clearly $\delta$ represents a phase shift (in the linear regime) between the perturbed density and electrostatic potential. For $\delta>0(\delta<0)$, the drift mode is linearly unstable (stable). More realistic models treat $\delta$ as a spatial operator. The one-field model (8) is valid in slab geometry $(\boldsymbol{\nabla} B=0)$. However, in a toroidal configuration, the magnetic field is inhomogeneous and additional terms due to curvature 
effects must be retained in the model equation (8).

In what follows we will concentrate on the equilibria and only allude to consequences on stability and transport as they arise. Specifically we will contrast tokamaks and stellarators by comparing key physical attributes of their equilibria. For the tokamak case we use an analytical expansion valid in the limit when the distance the long way around the torus is much longer than the distance the short way around the torus. For the stellarator case we use a full 3-dimensional numerically obtained equilibria for the TJ-II helical axis stellarator [22].

\subsection{Balancing the forces}

The conditions in today's fusion plasma are quite extreme with millionampere currents flowing in plasmas with magnetic fields of tens of thousands of gauss. The potential forces are enormous and uttermost care must be taken to balance these forces. In particular the magnetic forces and those due to the kinetic pressure need to balance in order to isolate the plasma, at a temperature of hundreds of million degrees, from the material walls.

Force balance between the internal forces of the kinetic pressure of the plasma and the forces of the external and internal magnetic fields and associated currents is normally studied using the magnetohydrodynamic model. In this

model the plasma is approximated as a perfectly conducting fluid. Balancing forces of the kinetic pressure with the forces of the magnetic field, we get

$$
c \nabla p=\mathbf{J} \times \mathbf{B} .
$$

Here $\mathbf{J}$ is the plasma current density, $\mathbf{B}$ is the total magnetic field and $p$ is the plasma pressure. $c$ is the speed of light. All the physical quantities given in this paper are in Centimetre-Gramme-Seconde (CGS) units. In general, equation(10), has to be solved numerically but in certain limits it is possible to obtain analytical results. One such case is shown in Fig.1. Fig.1 shows the magnitude of the magnetic field for a standard tokamak case. A portion of the torus has been artificially removed in order to observe the magnetic field strength variation as one moves across the plasma. Blue indicates the 
low field strength and red indicates the high field strength. For case shown in Fig.1, the magnetic forces balancing the kinetic pressure is mainly due to the large externally generated currents. The tokamak has rotational symmetry and each vertical cross section through this symmetry axis are identical. As a consequence the equilibria are essentially 2-dimensional. This leads to great simplification both of theoretical work and experimental work. Note that the magnetic field is stronger on the inside of the torus. The magnetic field strength varies roughly as $1 / R$, where $R$ is the distance from a point in the plasma to the revolution axis of the torus. For more details on tokamak equilibria, the reader is referred to Ref [26].

In a closed system a magnetic field line can behave in three distinct ways. It can close upon itself after a number of transits around the torus. It may trace out a surface, never closing upon itself. The surface thus traced out is consequently called a magnetic surface. The third possibility is when a magnetic field line fills a volume as it winds its way through the torus. Since particles and heat can travel quite freely along the magnetic field lines, such plasma configuration must be avoided as outward transport occurs by simply following the magnetic field lines. Thus, the existence magnetic surfaces, is an essential requirement for long-term confinement [27] The magnetic surfaces are also used to define the various types of curvilinear coordinates [40] invariably used in studies of equilibria. For general 3-dimensional equilibria, one can use the divergence-free condition for $\mathbf{B}$ to write the magnetic field in so-called straight-field line coordinates $[10,12,11]$

$$
\mathbf{B}=\nabla \alpha \times \nabla \psi,
$$

where $\alpha$ is the field line label and $2 \pi \psi$ is the enclosed poloidal magnetic flux. It is clear from equation (11) that the condition $\boldsymbol{\nabla} \cdot \mathbf{B}=0$ is automatically satisfied. Here $\psi$ is the radial coordinate which labels the magnetic surfaces. The field line label can be written as $\alpha=\zeta-q(\psi) \theta$, where $\zeta$ and $\theta$ are the so-called magnetic toroidal and poloidal angles, with period $2 \pi$, respectively. Finally, $q$ is the safety factor and this quantity is related to the pitch of the magnetic field line. Note that the representation (11) is not unique. For instance we note that the transformations $\alpha \mapsto \alpha+f(\psi)$ and $\psi \mapsto \psi+g(\alpha)$ leave the right-hand side of equation (11) (the actual physical quantity) unchanged. The representation (11) is convenient for analytical calculations. In practise, however, the curvilinear coordinates $\{\psi, \theta, \zeta\}$ have to be computed numerically, once the left-hand side of equation (11) has been specified. By 
construction, a magnetic field line $\alpha=$ const appears as a straight line in the $\theta-\zeta$ plane, with slope $q$. In straight-field line coordinates, a magnetic surface $\psi=$ const can be seen (in topological space) as a square with side $2 \pi$ in the poloidal and toroidal 'angles'. In real space, however, the magnetic surface is a fully 3 -dimensional object.

In Fig. 2 the coordinate grid for the tokamak case has been displayed. The shifted, concentric circles in this grid define the magnetic surfaces. The center of the toroidal coordinates in Fig. 2 is called the magnetic axis, an infinitelydegenerated magnetic surface which reduces to a circular line in the plasma center.

For comparison a top-view of the four-field period stellarator TJ-II [22] is shown in Fig.3. The physical quantity displayed is the magnetic field strength. The typical radius of the magnetic axis is $1 \mathrm{~m}$ and the average radius of the plasma is $0.2 \mathrm{~m}$. In Fig.4, the same magnetic surface as in Fig.3, but for a different viewing angle, is shown. The 'bumpy' structure of $B$ is due to the effects of the toroidal field coils. In contrast to tokamaks (Figs.1,2), stellarators (Figs.3,4) lack the continuous symmetry and require a full 3-dimensional consideration of equation (10). A low-resolution grid, defining a magnetic surface of TJ-II, is shown in Fig.5. In Fig.6, a cross section of TJ-II plasma is shown. Note the characteristic bean shape of the plasma. The hole in the is an artefact of the equilibrium code (The position of the magnetic axis is approximated using a polynomial expansion, which coefficients are determined from the neighboring magnetic surfaces). As stability is concerned, the plasma center is quite unimportant and crucial physics usually occur in outer regions of the confined plasma. While expansions are sometimes used for stellarators none is really very useful and the plasma geometry and the equilibrium magnetic field has to be determined numerically. The VMEC code $[41,42]$ developed at the Oak Ridge National Laboratory is a standard tool for finding stellarator equilibria. The VMEC code assumes the existence of nested magnetic surfaces $(\mathbf{B} \cdot \boldsymbol{\nabla} \psi \equiv 0)$ and uses an preconditioned steepest-decent method [42] to find the equilibrium solution. In this code, the magnetic field components for each magnetic surface are specified by Fourier series in curvilinear coordinates. There is some freedom in choosing the coordinates and in the VMEC code this is used to optimize the choice of one curvilinear coordinate (the poloidal 'angle') to accelerate the convergence. After the calculations we map the results back to a cylindrical grid which is optimum for display purposes. The final 'curvilinear' grid is shown 
in Fig.5.

\subsection{Magnetic Curvature}

The measured energy transport in magnetically confined plasmas is generally much larger than the predictions using particle trajectories and is often called anomalous for this reason $[4,13]$. The discrepancy is attributed to small scale plasma turbulence [14]. It is well known that small scale instabilities can cause enhanced transport in plasmas and they are major contenders to explain this difference. However, despite a considerable, world wide, effort over the last few decades the understanding of confinement is still relatively poor. As an unsatisfactory consequence, the next generation of large scale experiments are presently designed with the use of empirical or semi-empirical scaling laws $[13,15]$. Consequently, an increased understanding of transport of energy and particles across magnetic fields is of extreme importance.

The local structure of the magnetic field configuration plays an important role for the linear mode structure and stability of small-scale instabilities, and hence for energy transport, in magnetically confined plasmas. The magnetic field curvature is an example of distinct local attribute of a configuration. Curvature can either be locally favorable or unfavorable. Plasma instabilities are driven unstable by the magnetic curvature in much the same way as as heavy fluid is unstable on top of a light fluid. The plasma pressure gradients take on the role of gravity in the fluid example. A plasma configuration generally has regions of favorable curvature and regions of unfavorable curvature. Small scale instabilities tend to be localized in regions of unfavorable curvature while large scale instabilities tend to respond more to the average curvature.

The magnetic curvature is defined as

$$
\kappa \equiv\left(\mathbf{e}_{\|} \cdot \nabla\right) \mathbf{e}_{\|}
$$

where $\mathbf{e}_{\|} \equiv \mathbf{B} / B$ is a unit vector parallel to the equilibrium magnetic field. Curvature is an intrinsic quantity of closed magnetic systems such as stellarators and tokamaks. Favorable and unfavorable regions of curvature are closely related to the local plasma pressure gradient. If $\boldsymbol{\kappa} \cdot \boldsymbol{\nabla} p>0$, the magnetic curvature is unfavorable. For tokamaks, this occurs on the outside 
of the torus. On the inside of the torus $\boldsymbol{\kappa} \cdot \nabla p<0$, and the curvature is favorable. It is convenient to write the curvature vector in component form

$$
\boldsymbol{\kappa}=\kappa_{\mathrm{N}} \mathbf{n}+\kappa_{\mathrm{G}} \mathbf{b},
$$

where $\mathbf{n}$ is the unit normal vector, pointing outward of the magnetic surface. $\mathbf{b}$ is the unit binormal vector, which lies in the magnetic surface and is perpendicular to B. $\kappa_{\mathrm{N}}$ and $\kappa_{\mathrm{G}}$ are called normal and geodesic curvatures, respectively. In stellarator geometry, all the physical quantities (scalars and vectors) depend on all 3 coordinates of space. From the definition (12) we note that the curvature vector has no component parallel to B. Geodesic and normal curvatures usually appear in form of a linear combination in plasma stability theory. Other structural parameters of the confining magnetic field (see next section) are also present in the study of instabilities, and their specific forms depend on the theory used.

The components of the magnetic curvature, $\kappa_{\mathrm{N}}$ and $\kappa_{\mathrm{G}}$, can be determined analytically using techniques of curvilinear coordinates. For convenience, one can introduce a set of covariant basis vectors $\left\{\mathbf{e}_{\psi}, \mathbf{e}_{\theta}, \mathbf{e}_{\zeta}\right\}$, where $\mathbf{e}_{\mu} \equiv \partial \mathbf{r} / \partial \mu$, $\mathbf{r}$ is the local position vector in the plasma, and $\mu=\{\psi, \theta, \zeta\}$. Introducing the local Jacobian of the transformation, $\mathcal{J} \equiv[\boldsymbol{\nabla} \psi \cdot(\boldsymbol{\nabla} \theta \times \boldsymbol{\nabla} \zeta)]^{-1}$, one easily calculate the contravariant basis vectors, $\mathbf{e}^{\mu} \equiv \nabla \mu=\epsilon_{\mu \lambda \varphi} \mathcal{J}^{-1} \mathbf{e}_{\mu} \times \mathbf{e}_{\lambda}$, where $\epsilon_{\mu \lambda \varphi}$ is the usual Levi-Civita symbol for permutations. Then, one can decompose any vector $\mathbf{A}$ on the contravariant or covariant basis vectors. After some algebra one gets the geodesic curvature

$$
\kappa_{\mathrm{G}}=\frac{1}{\mathcal{J} \sqrt{g^{\psi \psi}}}\left[b_{\zeta} \kappa_{\theta}-b_{\theta} \kappa_{\zeta}\right]
$$

where

$$
\begin{aligned}
\kappa_{\theta} & \equiv\left(b^{\theta} \frac{\partial b^{\theta}}{\partial \theta}+b^{\zeta} \frac{\partial b^{\theta}}{\partial \zeta}\right) g_{\theta \theta}+\left(b^{\theta} \frac{\partial b^{\zeta}}{\partial \theta}+b^{\zeta} \frac{\partial b^{\zeta}}{\partial \zeta}\right) g_{\theta \zeta} \\
& +2 b^{\theta} b^{\zeta} \mathbf{e}_{\theta} \cdot \frac{\partial \mathbf{e}_{\theta}}{\partial \zeta}+\left(b^{\theta}\right)^{2} \mathbf{e}_{\theta} \cdot \frac{\partial \mathbf{e}_{\theta}}{\partial \theta}+\left(b^{\zeta}\right)^{2} \mathbf{e}_{\theta} \cdot \frac{\partial \mathbf{e}_{\zeta}}{\partial \zeta}
\end{aligned}
$$

and

$$
\begin{aligned}
\kappa_{\zeta} & \equiv\left(b^{\theta} \frac{\partial b^{\theta}}{\partial \theta}+b^{\zeta} \frac{\partial b^{\theta}}{\partial \zeta}\right) g_{\theta \zeta}+\left(b^{\theta} \frac{\partial b^{\zeta}}{\partial \theta}+b^{\zeta} \frac{\partial b^{\zeta}}{\partial \zeta}\right) g_{\zeta \zeta} \\
& +2 b^{\theta} b^{\zeta} \mathbf{e}_{\zeta} \cdot \frac{\partial \mathbf{e}_{\theta}}{\partial \zeta}+\left(b^{\theta}\right)^{2} \mathbf{e}_{\zeta} \cdot \frac{\partial \mathbf{e}_{\theta}}{\partial \theta}+\left(b^{\zeta}\right)^{2} \mathbf{e}_{\zeta} \cdot \frac{\partial \mathbf{e}_{\zeta}}{\partial \zeta}
\end{aligned}
$$


Similarly, it is easy to show that the normal component of the magnetic curvature is given by

$$
\kappa_{\mathrm{N}}=\frac{1}{\sqrt{g^{\psi \psi}}}\left[2 b^{\theta} b^{\zeta} \nabla s \cdot \frac{\partial \mathbf{e}_{\theta}}{\partial \zeta}+\left(b^{\theta}\right)^{2} \nabla s \cdot \frac{\partial \mathbf{e}_{\theta}}{\partial \theta}+\left(b^{\zeta}\right)^{2} \nabla s \cdot \frac{\partial \mathbf{e}_{\zeta}}{\partial \zeta}\right] .
$$

In equations $(14,17)$, we have introduced $b^{\theta} \equiv \mathbf{B} \cdot \boldsymbol{\nabla} \theta / B, b^{\zeta} \equiv \mathbf{B} \cdot \boldsymbol{\nabla} \zeta / B$, $B=\left(B_{\theta} B^{\theta}+B_{\zeta} B^{\zeta}\right)^{1 / 2}$ is the magnetic field strength, $g_{i j} \equiv \mathbf{e}_{i} \cdot \mathbf{e}_{j}$ and $g^{i j} \equiv \nabla i \cdot \nabla j$ are metric elements.

The effect of normal curvature and geodesic curvature in a plasma can be quite different. This is much the same way as a car turning at high speed on a free way responds differently to different form of curvatures. Consider the following situation. You are taking a turn on a freeway at high speed. If the surface of the road is not cambered the curvature is more or less geodesic, the centrifugal forces essentially acting in the surface and the friction between the road an your tyres is what saves you from disaster. If, on the other hand, the road is cambered we are changing the angle between normal vector of the road and the curvature. The curvature now has a large normal component. The car has a quite different and more secure response to this type of curvature provided, of course, that the road was cambered in the right direction.

The normal curvature for the tokamak and stellarator cases are shown in Fig.7 and Fig.8, respectively. For the tokamak case, the normal curvature vanishes on the top and the bottom of the torus. A negative normal curvature, which has been indicated in blue, occurs on the outside of the torus; a negative $\kappa_{\mathrm{N}}$ is destabilizing. Coming back to the analogy with the cambered freeway this region corresponds to the case when the road has been cambered the wrong way. For the stellarator case the corresponding negative normal curvature occurs on the outside of the bean shaped cross section. However, the direction changes as the bean shaped cross section twists around (Fig.8). Note the similarities between the tokamak and stellarator cases with the essential qualitative difference being that the negative region of curvature in the stellarator case twists itself around following the shape of the flux-surface. A more careful analysis reveals that the proportion of the region of favorable (positive) curvature is larger for the stellarator case.

The geodesic curvature for the tokamak and stellarator case are shown in 
Fig.9 and Fig.10, respectively. Regions colored in green correspond to a vanishing of the geodesic curvature. Negative geodesic curvature is indicated in blue while positive geodesic curvature is indicated in red. For low-frequency instabilities with elongated structure along the magnetic field line, the effect of the geodesic curvature is coupled to the normal component of $\boldsymbol{\kappa}$ and the local magnetic shear $[16,17]$.

\subsection{Local Magnetic Shear}

Bending the magnetic field lines costs energy and therefore the most dangerous instabilities in plasmas are often those that involve little or no bending of the magnetic field lines [18]. Magnetic shear is a measure of how the field lines changes directions as one moves from one magnetic surface to the next. Finite shear means that no instability of finite extent can exist without bending the magnetic field lines. Magnetic shear is therefore often stabilizing.

The local magnetic shear (LMS) is defined as [19]

$$
\mathcal{S} \equiv-\mathbf{s} \cdot \nabla \times \mathbf{s},
$$

where $\mathbf{s} \equiv(\boldsymbol{\nabla} \psi \times \mathbf{B}) /(\boldsymbol{\nabla} \psi \cdot \boldsymbol{\nabla} \psi)$ is a vector lying in the magnetic surface but perpendicular to magnetic field direction. As before, $\psi$ is the magnetic poloidal flux and it is used to label the magnetic surfaces. For a tokamak plasma with low kinetic pressure, the LMS can be calculated using an expansion technique [21, 20]. For the stellarator case, the explicit form of the LMS (18) can be written in straight-field line coordinates. After lengthy calculations, we get

$$
\begin{aligned}
\mathcal{S} & =C\left\{( B _ { \zeta } g _ { s \theta } - B _ { \theta } g _ { s \zeta } ) \left[g_{\theta \theta} \frac{\partial B_{\zeta}}{\partial \zeta}-g_{\theta \zeta}\left(\frac{\partial B_{\theta}}{\partial \zeta}+\frac{\partial B_{\zeta}}{\partial \theta}\right)+g_{\zeta \zeta} \frac{\partial B_{\theta}}{\partial \theta}\right.\right. \\
& +B_{\zeta}\left(\frac{\partial g_{\theta \theta}}{\partial \zeta}-\frac{\partial g_{\theta \zeta}}{\partial \theta}\right)+B_{\theta}\left(\frac{\partial g_{\zeta \zeta}}{\partial \theta}-\frac{\partial g_{\theta \zeta}}{\partial \zeta}\right) \\
& +\left(B_{\theta} g_{\theta \zeta}-B_{\zeta} g_{\theta \theta}\right)\left(\frac{1}{\mathcal{J}} \frac{\partial \mathcal{J}}{\partial \zeta}+\frac{1}{\sqrt{g^{s s}}} \frac{\partial \sqrt{g^{s s}}}{\partial \zeta}+\frac{1}{B} \frac{\partial B}{\partial \zeta}\right) \\
& \left.+\left(B_{\zeta} g_{\theta \zeta}-B_{\theta} g_{\zeta \zeta}\right)\left(\frac{1}{\mathcal{J}} \frac{\partial \mathcal{J}}{\partial \theta}+\frac{1}{\sqrt{g^{s s}}} \frac{\partial \sqrt{g^{s s}}}{\partial \theta}+\frac{1}{B} \frac{\partial B}{\partial \theta}\right)\right] \\
& +\left(B_{\zeta} g_{\theta \theta}-B_{\theta} g_{\theta \zeta}\right)\left[g_{\theta \zeta} \frac{\partial B_{\zeta}}{\partial s}-g_{\zeta \zeta} \frac{\partial B_{\theta}}{\partial s}-g_{s \theta} \frac{\partial B_{\zeta}}{\partial \zeta}+g_{s \zeta} \frac{\partial B_{\theta}}{\partial \zeta}\right.
\end{aligned}
$$




$$
\begin{aligned}
& +B_{\zeta}\left(\frac{\partial g_{\theta \zeta}}{\partial s}-\frac{\partial g_{s \theta}}{\partial \zeta}\right)+B_{\theta}\left(\frac{\partial g_{s \zeta}}{\partial \zeta}-\frac{\partial g_{\zeta \zeta}}{\partial s}\right) \\
& +\left(B_{\theta} g_{\zeta \zeta}-B_{\zeta} g_{\theta \zeta}\right)\left(\frac{1}{\mathcal{J}} \frac{\partial \mathcal{J}}{\partial s}+\frac{1}{\sqrt{g^{s s}}} \frac{\partial \sqrt{g^{s s}}}{\partial s}+\frac{1}{B} \frac{\partial B}{\partial s}\right) \\
& \left.+\left(B_{\zeta} g_{s \theta}-B_{\theta} g_{s \zeta}\right)\left(\frac{1}{\mathcal{J}} \frac{\partial \mathcal{J}}{\partial \zeta}+\frac{1}{\sqrt{g^{s s}}} \frac{\partial \sqrt{g^{s s}}}{\partial \zeta}+\frac{1}{B} \frac{\partial B}{\partial \zeta}\right)\right] \\
& +\left(B_{\zeta} g_{\theta \zeta}-B_{\theta} g_{\zeta \zeta}\right)\left[g_{s \theta} \frac{\partial B_{\zeta}}{\partial \theta}-g_{s \zeta} \frac{\partial B_{\theta}}{\partial \theta}-g_{\theta \theta} \frac{\partial B_{\zeta}}{\partial s}+g_{\theta \zeta} \frac{\partial B_{\theta}}{\partial s}\right. \\
& +B_{\zeta}\left(\frac{\partial g_{s \theta}}{\partial \theta}-\frac{\partial g_{\theta \theta}}{\partial s}\right)+B_{\theta}\left(\frac{\partial g_{\theta \zeta}}{\partial s}-\frac{\partial g_{s \zeta}}{\partial \theta}\right) \\
& +\left(B_{\theta} g_{s \zeta}-B_{\zeta} g_{s \theta}\right)\left(\frac{1}{\mathcal{J}} \frac{\partial \mathcal{J}}{\partial \theta}+\frac{1}{\sqrt{g^{s s}}} \frac{\partial \sqrt{g^{s s}}}{\partial \theta}+\frac{1}{B} \frac{\partial B}{\partial \theta}\right) \\
& \left.\left.+\quad\left(B_{\zeta} g_{\theta \theta}-B_{\theta} g_{\theta \zeta}\right)\left(\frac{1}{\mathcal{J}} \frac{\partial \mathcal{J}}{\partial s}+\frac{1}{\sqrt{g^{s s}}} \frac{\partial \sqrt{g^{s s}}}{\partial s}+\frac{1}{B} \frac{\partial B}{\partial s}\right)\right]\right\}
\end{aligned}
$$

where

$$
C \equiv\left(\frac{1}{\dot{\psi} g^{s s} \mathcal{J}^{3 / 2}}\right)^{2} .
$$

Of course, the calculations for the LMS are performed numerically.

The local magnetic for the tokamak and stellarator cases are shown in Fig.9 and Fig.10 respectively. Now we can clearly see distinct differences between the tokamak and stellarators. For the tokamak case [21], dark blue indicates zero shear and red indicates large positive shear and the shear is seen to be smoothly varying and in fact, to first approximation, constant on a magnetic surface. This is contrasted with the stellarator case where blue indicates large negative values and red indicates large positive values. Here the shear is rapidly varying with a surface average close to zero.

\section{Visualization}

Advanced Visual Systems Inc (AVS) [44] has developed a powerful, user friendly method for displaying scientific results. It has a wide range of applications in physics, chemistry, biology and other areas of science and engineering. AVS graphic techniques allow the user to read, manipulate and display numerical data. The AVS input data file usually contains one, two or 
three space coordinates (physical location of a grid point) and one or more physical quantities (such as density, temperature, fluid velocity) associated with a given set of grid points. AVS system could handle scalar as well as vectorial quantities.

The graphics presented in the previous sections were created using the AVS [44] visualization techniques. Here we will briefly indicate what is typically involved in this process. We will use the TJ-II helical axis stellarator as an example for the discussion in this section. Manipulation of the AVS input data file is done through an organized order called a network. A network is made of a group of modules, each of them manipulating the data in a specified way. The user connects a set of existing modules or develop new ones as the needs develops.

As we already pointed out in the previous sections, the confined plasmas in toroidal fusion machines can be seen as a set of nested doughnut-like surfaces (with various amount of twisting, depending on the configuration), the so-called magnetic surfaces. The actual details of each magnetic surface depends on the machine topology (tokamak or stellarator) and on the plasma parameters (such as the current flowing in the plasma, the plasma density and temperature, etc.).

A magnetic surface can be topologically represented as a square of side $2 \pi$ in $[\theta, \zeta]$ domain where $\theta$ and $\zeta$ are some (often curvilinear) angle-like variables. $\theta$ indicates the position as one moves the short way around the torus, while $\zeta$ indicates the position as one moves the long way around the torus. Usually, for sake of simplicity, stability calculations are carried out in this 'curvilinear (or topological) space'. However, when one has to compare theoretical studies and experimental results or simply to gain some physical insight about the geometrical aspects of the problem, one has to display the magnetic surface as it appears in our standard 3-dimensional space. The transformation from topological space to 'real' 3-dimensional space (Fig.13) is called mapping and it is discussed below.

As it happens, in the output from the equilibrium code VMEC [42], $\zeta=\phi$, where $\phi$ is the usual azimuthal angle in cylindrical coordinates. The VMEC code also outputs the cylindrical coordinates $R$ and $Z$ in terms of Fourier series. These components together with the dependence on the cylindrical 
angle $\phi$ completely specify a given magnetic surface

$$
\begin{aligned}
& R(\theta, \phi)=\sum_{m=0, n=-N}^{m=M, n=+N} R_{m n} \cos \left(m \theta+N_{\text {per }} n \phi\right), \\
& Z(\theta, \phi)=\sum_{m=0, n=-N}^{m=M, n=+N} Z_{m n} \sin \left(m \theta+N_{\text {per }} n \phi\right) .
\end{aligned}
$$

Here $N_{\text {per }}$ is the number of field periods or the degree of discrete symmetry. The stellarator presented in this paper has four-fold symmetry (Fig.3) and $N_{\text {per }}=4$. The magnetic field components are also given in terms of Fourier series, similar to equations $(21,22)$. The required resolution generally depends on the object and the quantities being displayed. The stellarator considered here has a fairly detailed structure requiring a relatively large number of Fourier components. For the figures displayed we used $M=7$ and $N=14$. A uniform grid was used in $\theta$ and $\phi$ with large enough number of grid points $N_{\theta}$ and $N_{\phi}$ to satisfy $N_{\theta} \gg M$ and $N_{\phi} \gg N$. Computational experience told us that a topological grid with $N_{\theta}=100$ and $N_{\phi}=600$ provides the required resolution when displayed in 3-dimensional space. Unphysical oscillations were observed for smaller values of $N_{\theta}$ and $N_{\phi}$. We have checked that for greater values of these parameters the resolution of the end product was left unchanged.

The basic procedure of the mapping technique is as follows. In the first stage, a uniform grid is created in topological $[\theta, \phi]$ space and meaningful physical quantities to be displayed are calculated on this grid (Fig.13). Then, using the VMEC representation for cylindrical coordinates given above, Cartesian coordinates of each grid point are computed. Writing $\theta_{i}=i \Delta \theta$ and $\phi_{j}=$ $j \Delta \phi$ where $i$ and $j$ are integers, a point on a given magnetic surface is given by

$$
\begin{gathered}
x_{i j}=R_{i j} \cos \theta_{i j} \\
y_{i j}=R_{i j} \sin \theta_{i j} \\
z_{i j}=Z_{i j}
\end{gathered}
$$

where $R_{i j} \equiv R\left(\theta_{i}, \phi_{j}\right)$ and $Z_{i j} \equiv Z\left(\theta_{i}, \phi_{j}\right)$ are calculated using equations $(21,22)$. Using equations $(23,24,25)$, the position vector $\mathbf{r}=x \widehat{\mathbf{x}}+y \widehat{\mathbf{y}}+z \widehat{\mathbf{Z}}$ is known everywhere on the magnetic surface. Then one can evaluate all the quantities required in equations $(17,14,19)$ by calculating the following quantities (in that order) 
- Covariant basis vectors, $\mathbf{e}_{\mu}=\partial \mathbf{r} / \partial \mu$ for $\mu=\{\psi, \theta, \zeta\}$;

- Covariant metric elements, $g_{\mu \lambda}=\mathbf{e}_{\mu} \cdot \mathbf{e}_{\lambda}$ for $(\mu, \lambda)=\{\psi, \theta, \zeta\}$;

- Jacobian of the transformation, $\mathcal{J}=\mathbf{e}_{\psi} \cdot\left(\mathbf{e}_{\theta} \times \mathbf{e}_{\zeta}\right)$;

- Contravariant basis vectors, $\mathbf{e}^{\mu}=\epsilon_{\mu \lambda \varphi} \mathcal{J}^{-1}\left(\mathbf{e}_{\lambda} \times \mathbf{e}_{\varphi}\right)$ for $(\mu, \lambda, \varphi)=$ $\{\psi, \theta, \zeta\}$

- Contravariant metric elements, $g^{\mu \lambda}=\mathbf{e}^{\mu} \cdot \mathbf{e}^{\lambda}$ for $(\mu, \lambda)=\{\psi, \theta, \zeta\}$.

The AVS input data file, the first module in the network hierarchy, is constructed using quadrahedron cells made of four nodes $\left(x_{i j}, y_{i j}, z_{i j}\right)$ given above. Note that a surface element $\Delta \theta \Delta \phi$ in topological space on a given magnetic surface corresponds to a twisted surface element in 3-Dimensional space. This is illustrated in Fig.13.

The AVS input file requires information on how grid points are connected together. When grid points fill a volume in a smooth, well-behaved way, an AVS module can create appropriate connections automatically. However, in our case, even though the grid is uniform in $[\theta, \phi]$ space, grid points are distributed in a non-trivial way in real space (Fig.5). This form of data is called Unstructured Cell Data (UCD) [44]. An example of the grid was shown in Fig.2 (tokamak case) and Fig.5 (stellarator case). Care must be taken to ensure appropriate torus periodicity, For instance, any physical quantity $F$ must satisfy the requirement of poloidal periodicity, $F(\theta, \zeta)=F(\theta+2 \pi, \zeta)$, and toroidal periodicity, $F(\theta, \zeta)=F(\theta, \zeta+2 \pi)$.

\section{Summary}

Advanced visualization techniques have been shown to be extremely useful in modern plasma physics. Physical insight can be gained in the understanding of complicated 3-dimensional magnetic topologies.

Because of long-range, collective-type phenomena, the physic of magnetically confined plasmas is rather involved. In section 2, we have presented the four basic areas in the study of controlled thermo-nuclear fusion; these are: equilibria, stability, transport and heating. Complete understanding of each of these research areas is a formidable task in itself. Ultimately, a global understanding of magnetically confined plasmas would be required. 


\section{Acknowledgments}

Fruitful discussions with H. Kanugo are greatly acknowledged. The author was supported by a Natural Sciences and Engineering Research Council of Canada (NSERC) Research Fellowship.

\section{References}

[1] R.E. Post, Scientific American, p. 3, 1957.

[2] L. Spitzer, Physics of Fluids, 1, p. 253, 1958.

[3] S.I. Braginskii, Review of Plasma Physics, Vol 1, Consultant Bureau (M.A. Leontovitch Ed), New York, 1965.

[4] P.C. Liewer, Nuclear Fusion, 25(5), p. 543, 1985.

[5] F. Wagner and U. Stroth, Plasma Physics and Controled Fusion, 35, p. 1321, 1993.

[6] A. Hasegawa and K. Mima, Physical Review Letters, 39(4), p. 205, 1977.

[7] W. Horton, Basic Plasma Physics, Vol. 2, Elsevier Science (M.N. Rosenbluth and R.Z. Sagdeev Eds), 1984.

[8] W. Horton, Physics of Fluids, B1(3), p. 524, 1989.

[9] P. Terry and W. Horton, Physics of Fluids, 25(3), p. 491, 1982.

[10] M.D. Kruskal and R.M. Kulsrud, Physics of Fluids 1(4), p. 265, 1958.

[11] J.M. Greene, J.L. Johnson, M.D. Kruskal and L. Wilets, Physics of Fluids, 5(9), p. 1063, 1962.

[12] J.M. Greene and J.L. Johnson, Physics of Fluids 5(5)m p. 510, 1962.

[13] J.D. Callen, Physics Fluids, B4(7), p. 2142, 1992.

[14] W.M. Tang, Nuclear Fusion, 18(8), p. 1089, 1978. 
[15] W.A. Houlberg, D.W. Ross, G. Bateman, S.C. Cowley, P.C. Efthimion, W.W. Pfeiffer, G.D. Porter, D.E. Shumaker, L.E. Sugiyama and J.C. Wiley, Physics of Fluids, B2(12), p. 2913, 1990.

[16] J.L.V. Lewandowski, Canadian Journal of Physics, 75, p. 891, 1997.

[17] J.L.V. Lewandowski, Plasma Physics and Controlled Fusion, 40(2), p. 283, 1998.

[18] J.M. Greene and M.S. Chance, Nuclear Fusion, 21(4), p. 453, 1981.

[19] J.M. Greene and J.L. Johnson, Plasma Physics, 10, p. 729, 1968.

[20] J.L.V. Lewandowski and M. Persson, Australian Journal of Physics, 48(3), p. 126, 1996.

[21] J.L.V. Lewandowski and M. Persson, Plasma Physics and Controlled Fusion, 37, p. 1199, 1995.

[22] C. Alejaldre, J.J.A. Gozalo, J.B. Perez, F.C. Magana, J.R.C. Diaz, J.G. Perez, A. Lopez-Fraguas, L. Garcia, V.I. Krivienski, R. Martin, A.P. Navarro, A. Perea A. Rodringuez-Yunta, M.S. Ayza and A. Varias, TJII project: a flexiable heliac stellarator, Fusion Technology, 17 p. 131, 1990.

[23] J.B. Adams, A review of nuclear fusion research, Proceedings of the Physical Society, Vol. 89, pp. 189-216 (1966).

[24] J. Wesson, Tokamaks, Clarendon Press (Oxford), 1987.

[25] L. Spitzer, The stellarator, Scientific American, pp. 2-9 (October 1958)

[26] R.B. White, Theory of tokamak plasmas North-Holland Physics, Amsterdam, 1989

[27] H. Grad, Toroidal containment of a plasma, Physics of Fluids, 10(1), p. 137, 1967.

[28] C.Z. Cheng and H. Okuda, Journal of Computational Physics, 25, p. $133,1977$.

[29] C.Z. Cheng and H. Okuda, Nuclear Fusion, 18, p. 587, 1978. 
[30] W.W. Lee and H. Okuda, Journal of Computational Physics, 26, p. 139, 1978.

[31] W.W. Lee, Physics of Fluids, 26, p. 556, 1983.

[32] W.W. Lee, Gyrokinetic Particle Simulation Model, Journal of Computational Physics, 72, p. 243, 1987.

[33] J.C. Adam and A. Gourdin Serveniere and A.B. Langdon, Journal of Computational Physics, 47, p. 229, 1982.

[34] A.M. Dimits and W.W. Lee, Partially linearized algorithms in gyrokinetic particle simulations, Technical Report PPPL-2718. Plasma Physics Laboratory, Princeton University.

[35] G. DiPeso and E.C. Morse and R.W. Ziolkowski, Journal of Computational Physics, 96, p. 325, 1991.

[36] M. Kotschenreuther, Proceedings of the $13^{\text {th }}$ International Conference on Plasma Physics and Controlled Nuclear Fusion Research, Vol 2 (Vienna: IAEA), p. 361, 1991.

[37] S.E. Parker and W.W. Lee, Physics of Fluids, B5, p. 77, 1993.

[38] T. Tajima, Computational Plasma Physics with applications to fusion and astrophysics, Addison Wesley, New York, 1989.

[39] M.J. LeBrun and T. Tajima, Particle simulation in curvilinear coordinate systems, Technical Report IFSR 374, Institure for Fusion Studies, Texas University.

[40] W.D D'Haeseleer, W.N. Hitchon, J.D. Callen and J.L. Shohet, Flux coordinates and magnetic field structure, Springer series in computational physics, Berlin, 1991.

[41] S.P. Hirshman and J.T. Hogan, Journal of Computational Physics, 63, p. 329, 1986.

[42] Hirshman S.P. and Betancourt, O., Preconditioned decent algorithm for rapid calculations of magnetohydrodynamic equilibria, Journal of Computational Physics, Vol. 96, 1991, pp. 99-109. 
[43] J.M. Greene and J.L. Johnson, Interchange instabilities in ideal hydromagnetic theory, Plasma Physics, Vol. 10, pp. 729-745 (1962).

[44] AVS User's guide, Advanced Visual Sytems Inc., Waltham, MA 02154, USA, copyrigth 1992. 


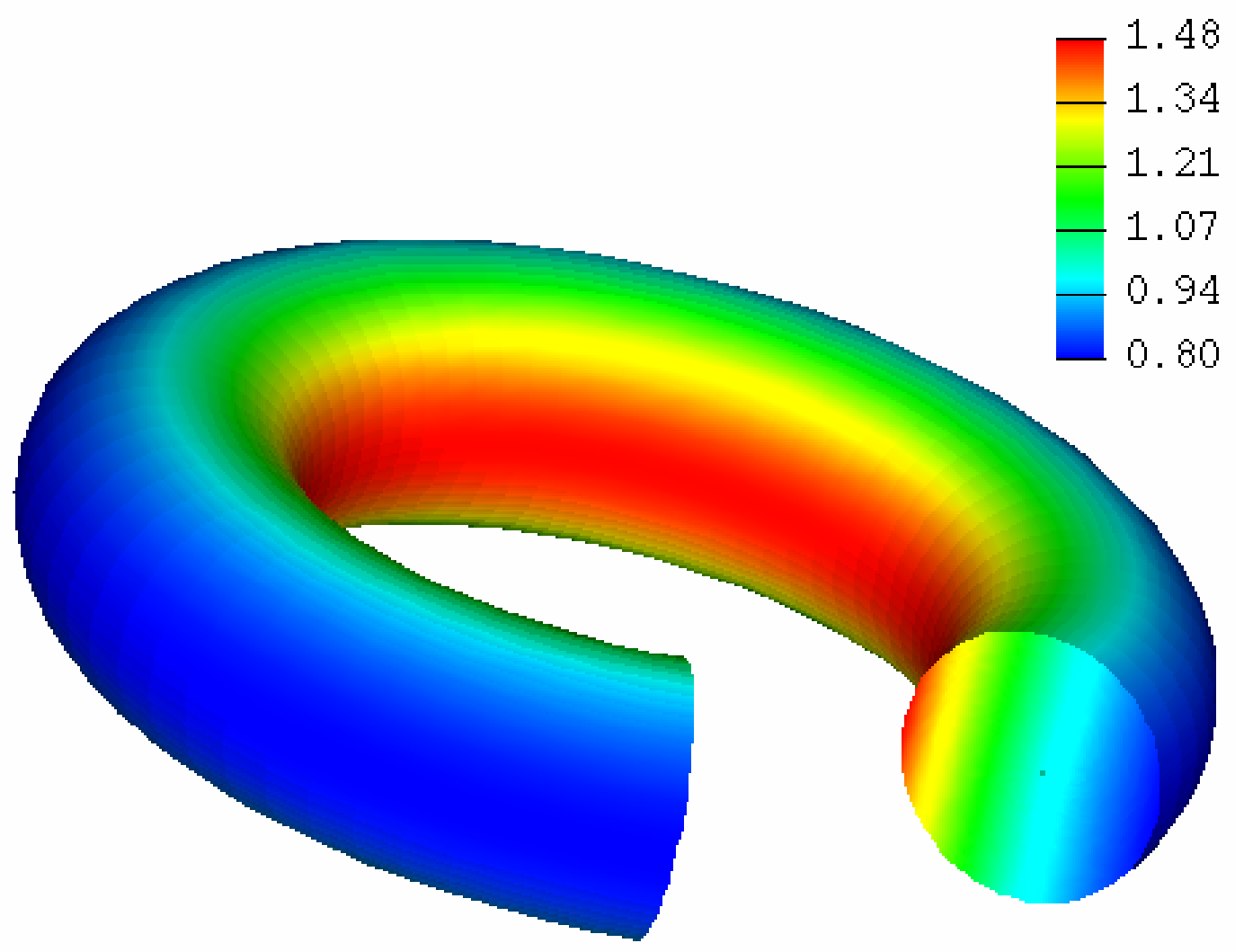

Figure 1: The magnetic field strength for the standard, axi-symmetric tokamak. Red region corresponds to high magnetic field while blue region indicates low magnetic field. 


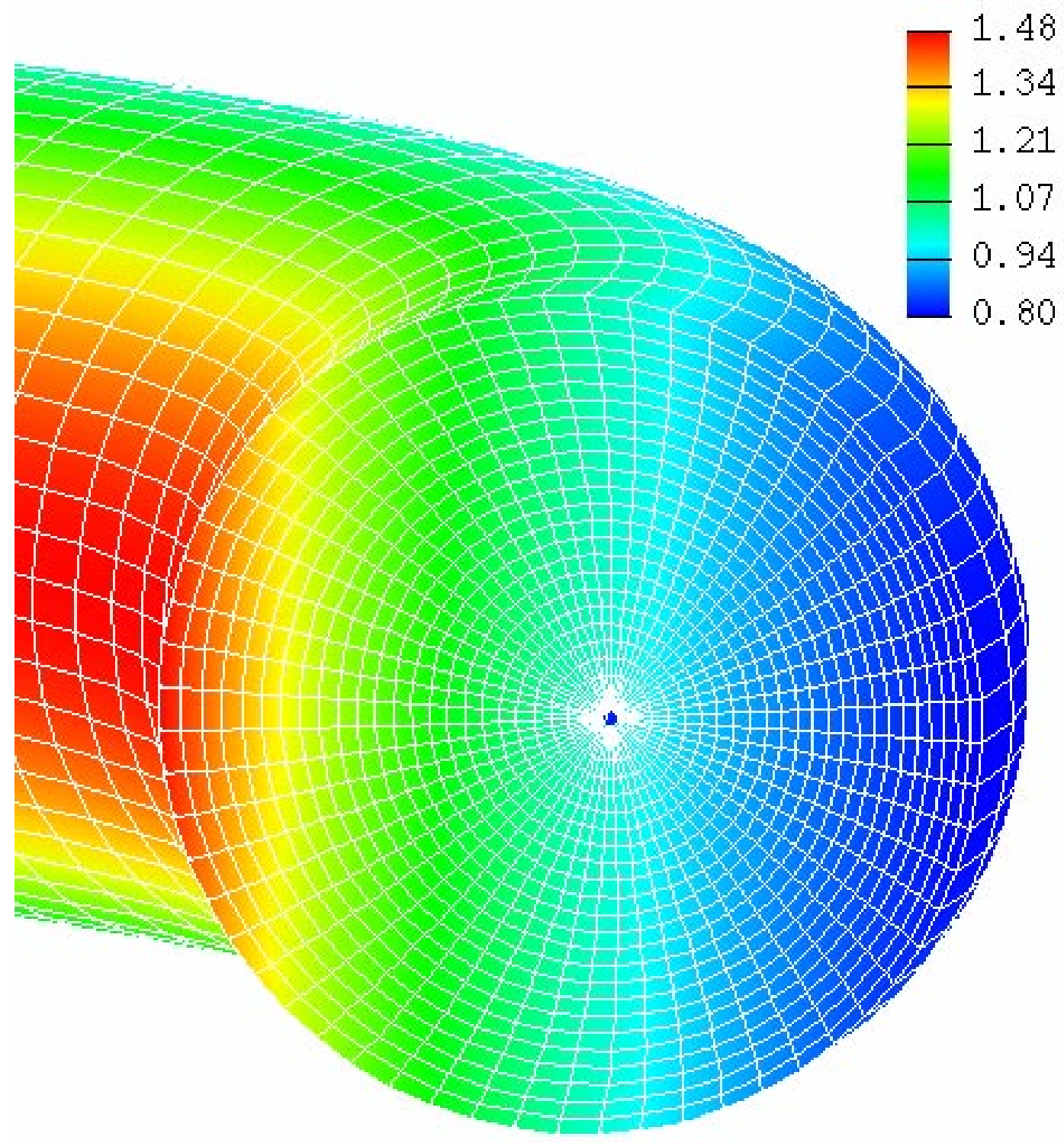

Figure 2: Curvilinear coordinate grid for the standard tokamak. The shifted circles are the magnetic surfaces. The amount of shift is proportional to the ratio of the plasma potential energy to the magnetic energy. 


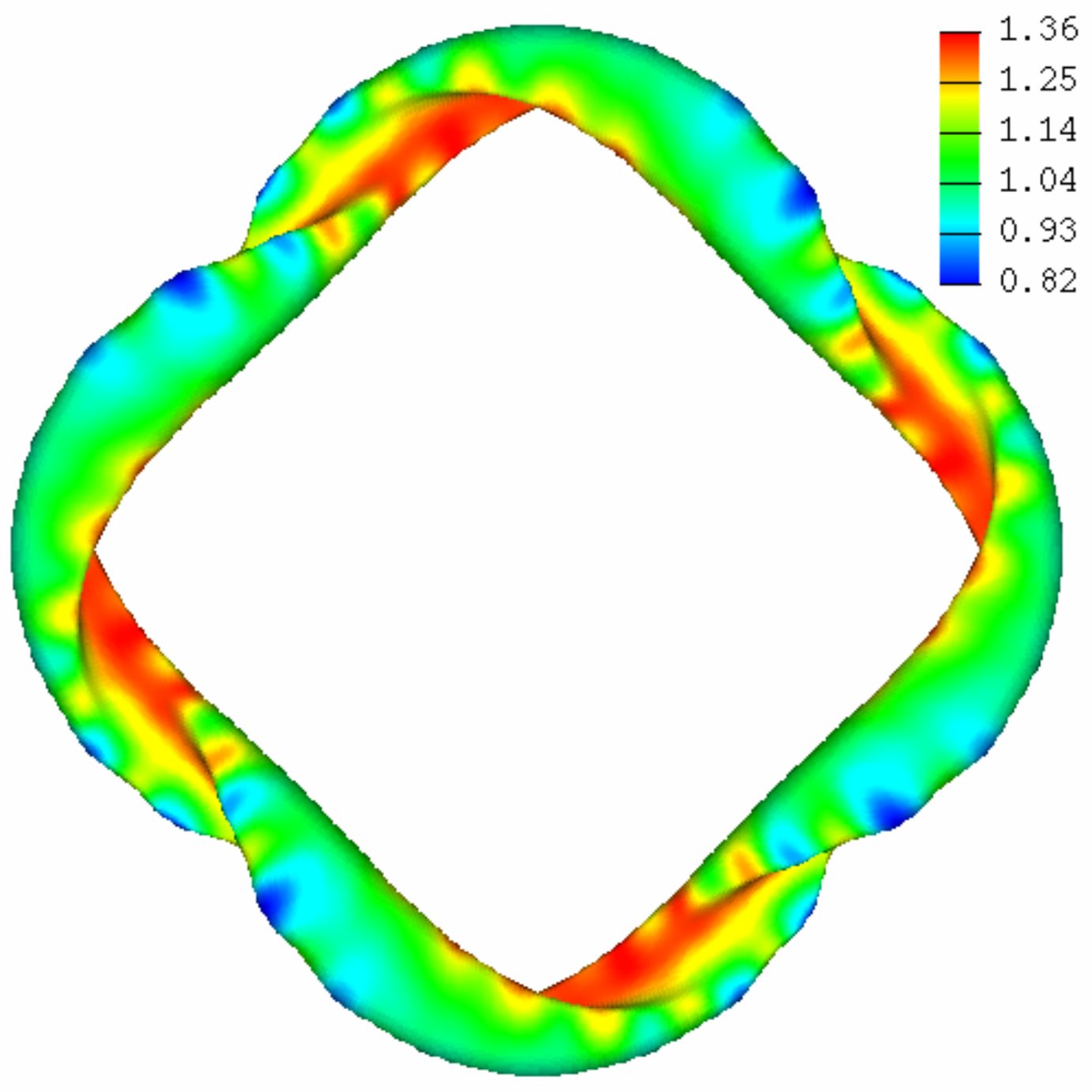

Figure 3: Top-view of the 4-field period stellarator TJ-II [22]. The magnetic field strength is shown. We note the detailed structure of $B$ due to the effects of the toroidal field coils. 


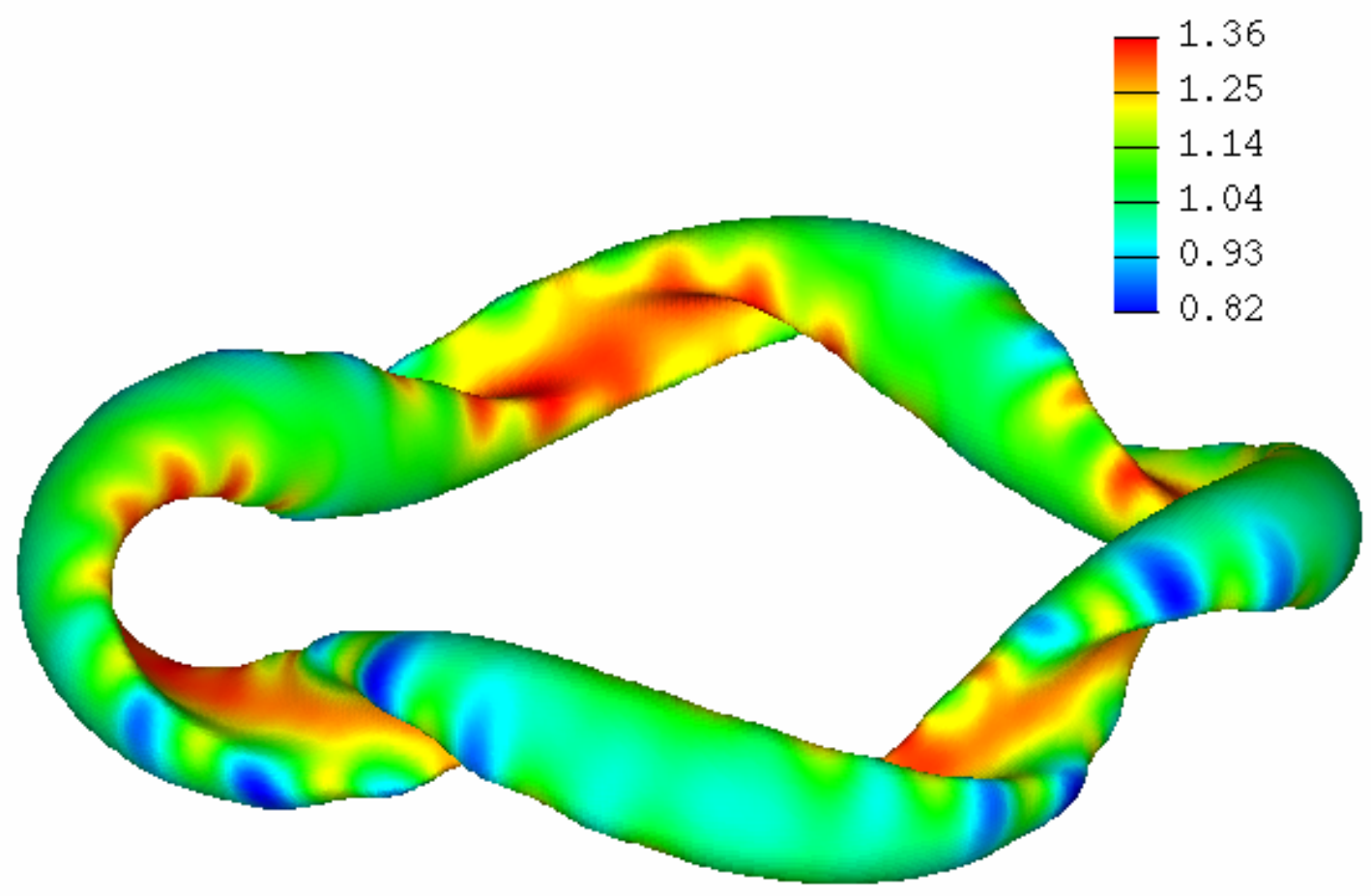

Figure 4: Magnetic field strength on a magnetic surface of stellarator TJII [22]. The helical structure of the magnetic axis is visible. 


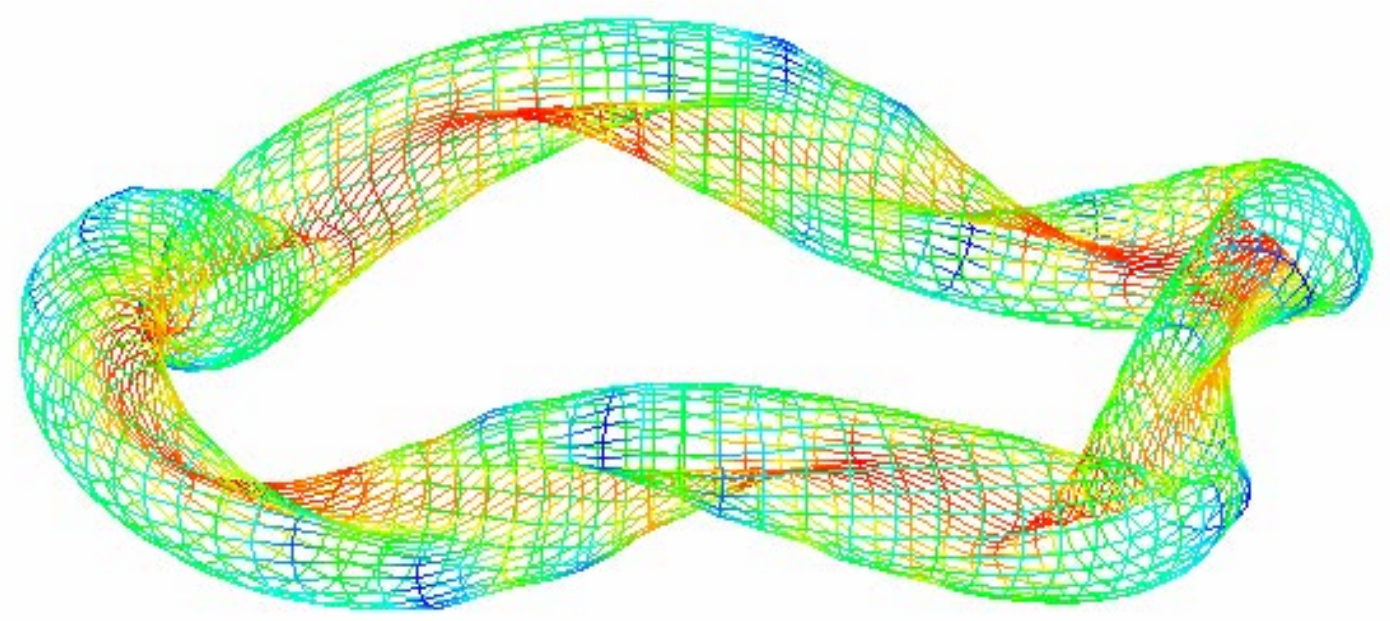

Figure 5: Low-resolution grid of a magnetic surface of stellarator TJ-II [22]. Each intersection between two lines defines a node, whereas each twisted parallelogram (made of 4 nodes) defines a cell. 


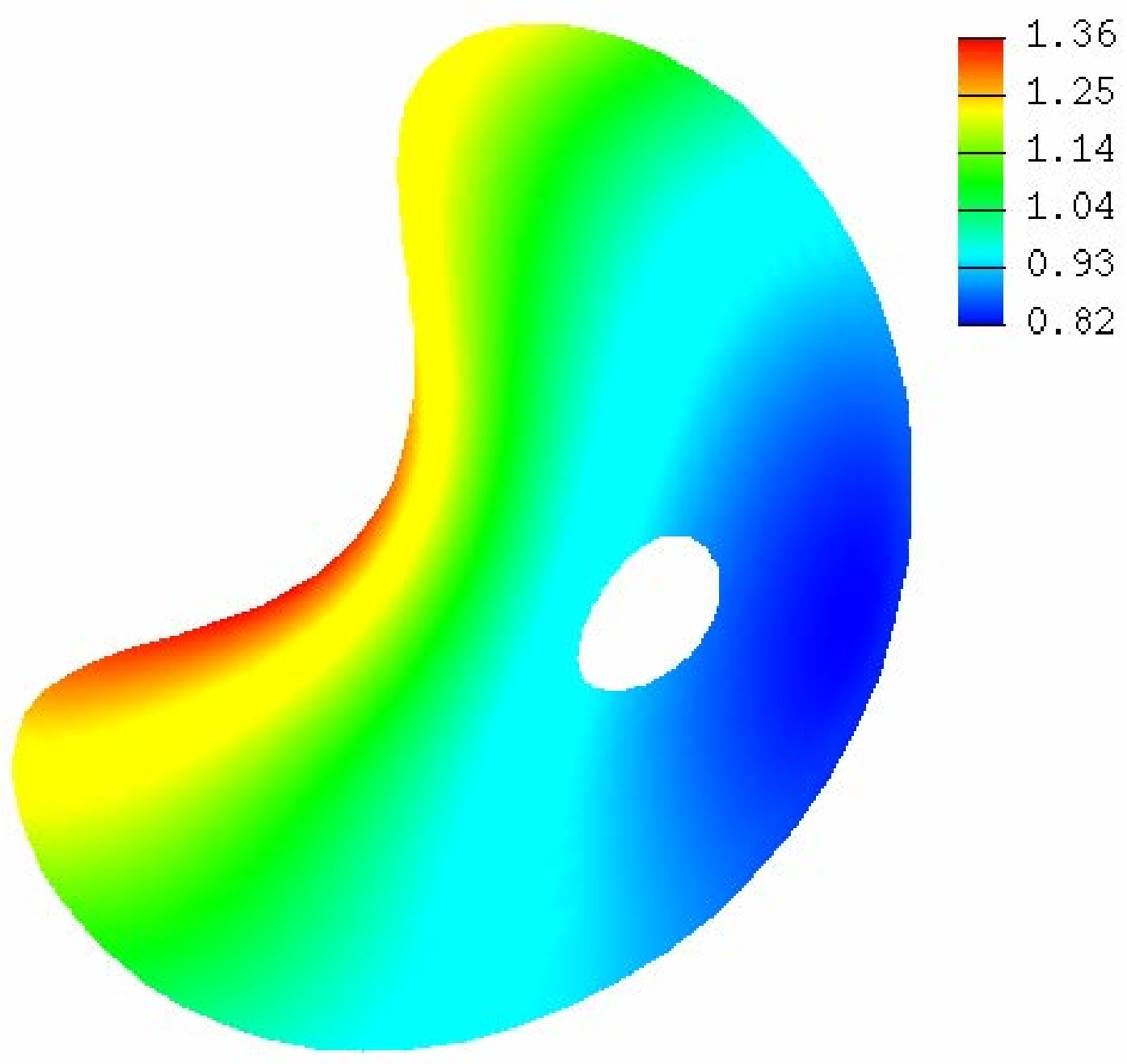

Figure 6: Magnetic field strength in the cross section of TJ-II [22] stellarator plasma. The bean-shaped cross section twists around as we move the long way around the torus. The 'hole' is an artefact of the VMEC code (see main text). 


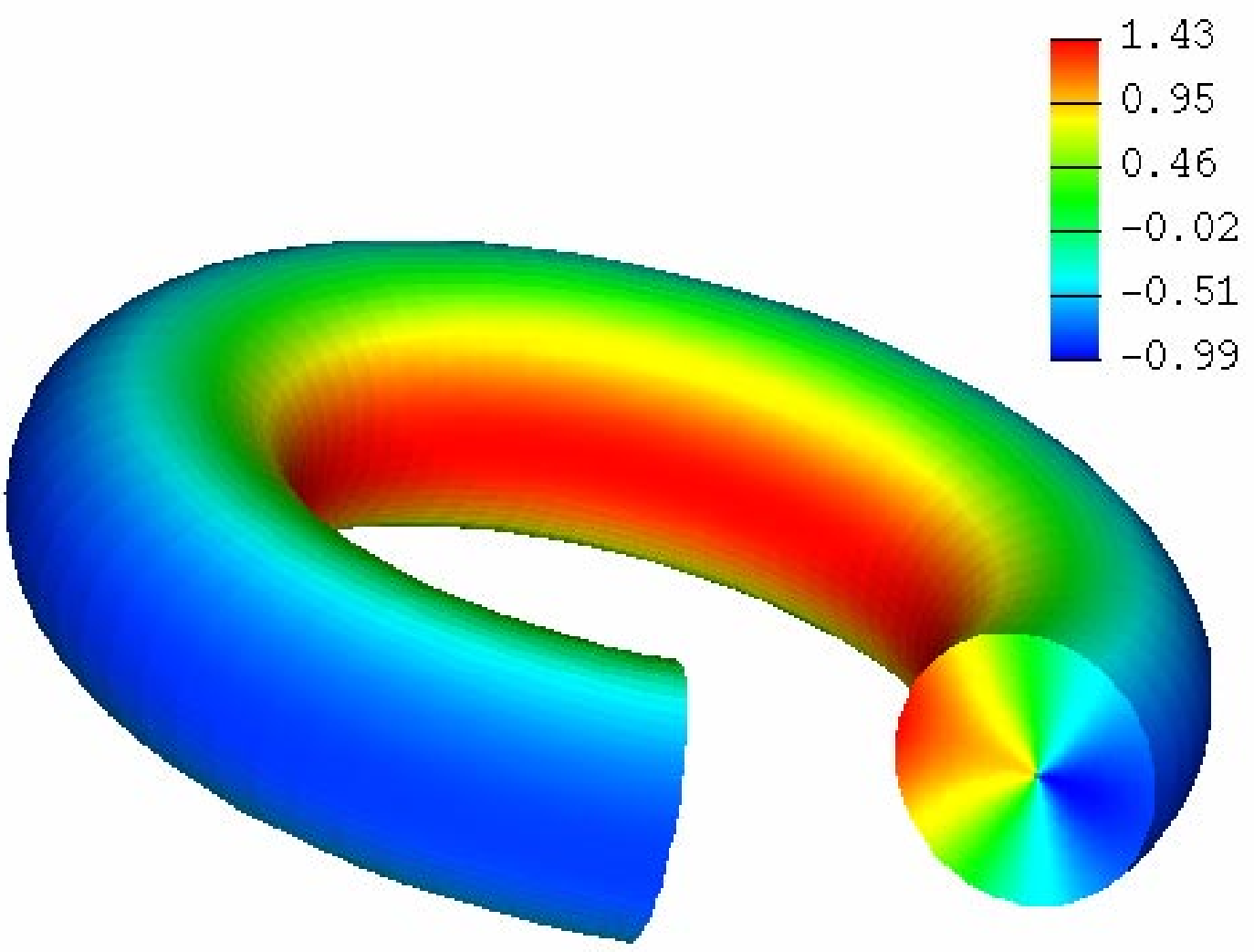

Figure 7: Normal magnetic curvature for the standard tokamak. The region of unfavorable (negative) curvature is indicated in blue. Favorable (positive) curvature is shown in red. 


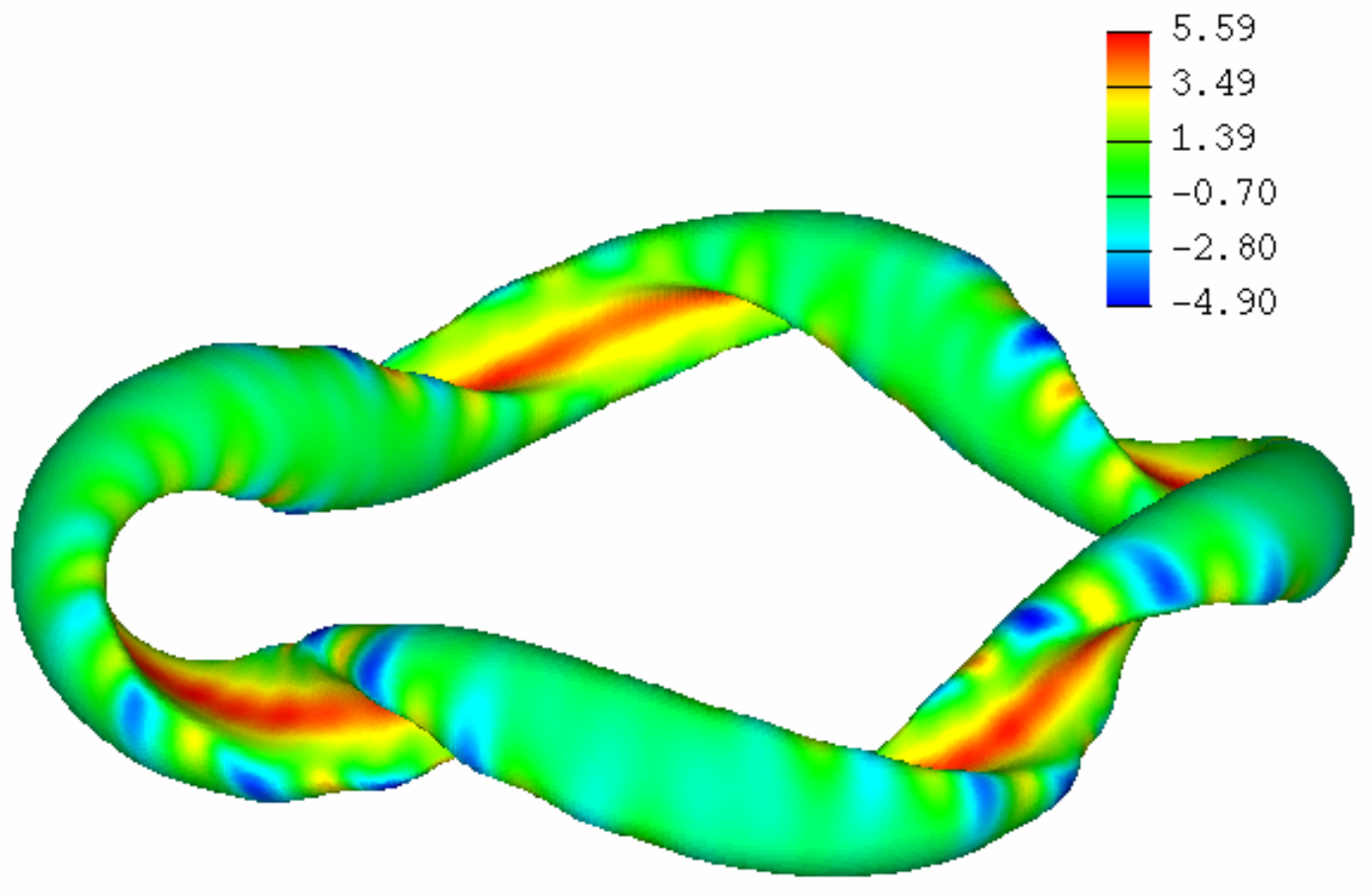

Figure 8: Normal magnetic curvature on a magnetic surface of stellarator TJ-II [22]. The region of unfavorable curvature is indicated in blue and it twists around the long way along the torus. 


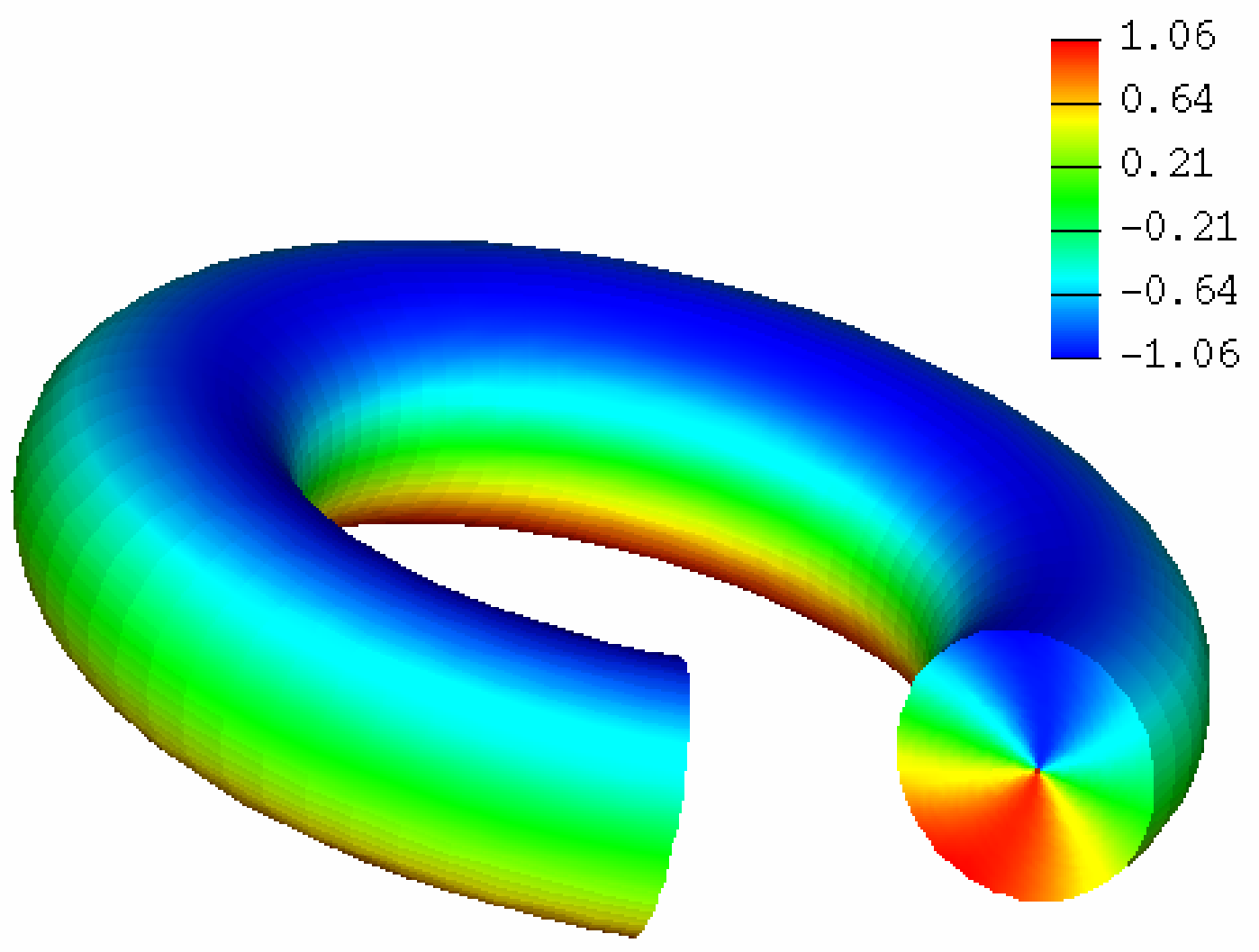

Figure 9: Geodesic magnetic curvature for the standard tokamak. The vanishing of the geodesic curvature, indicated in green, occurs at the plasma inside and outside. 


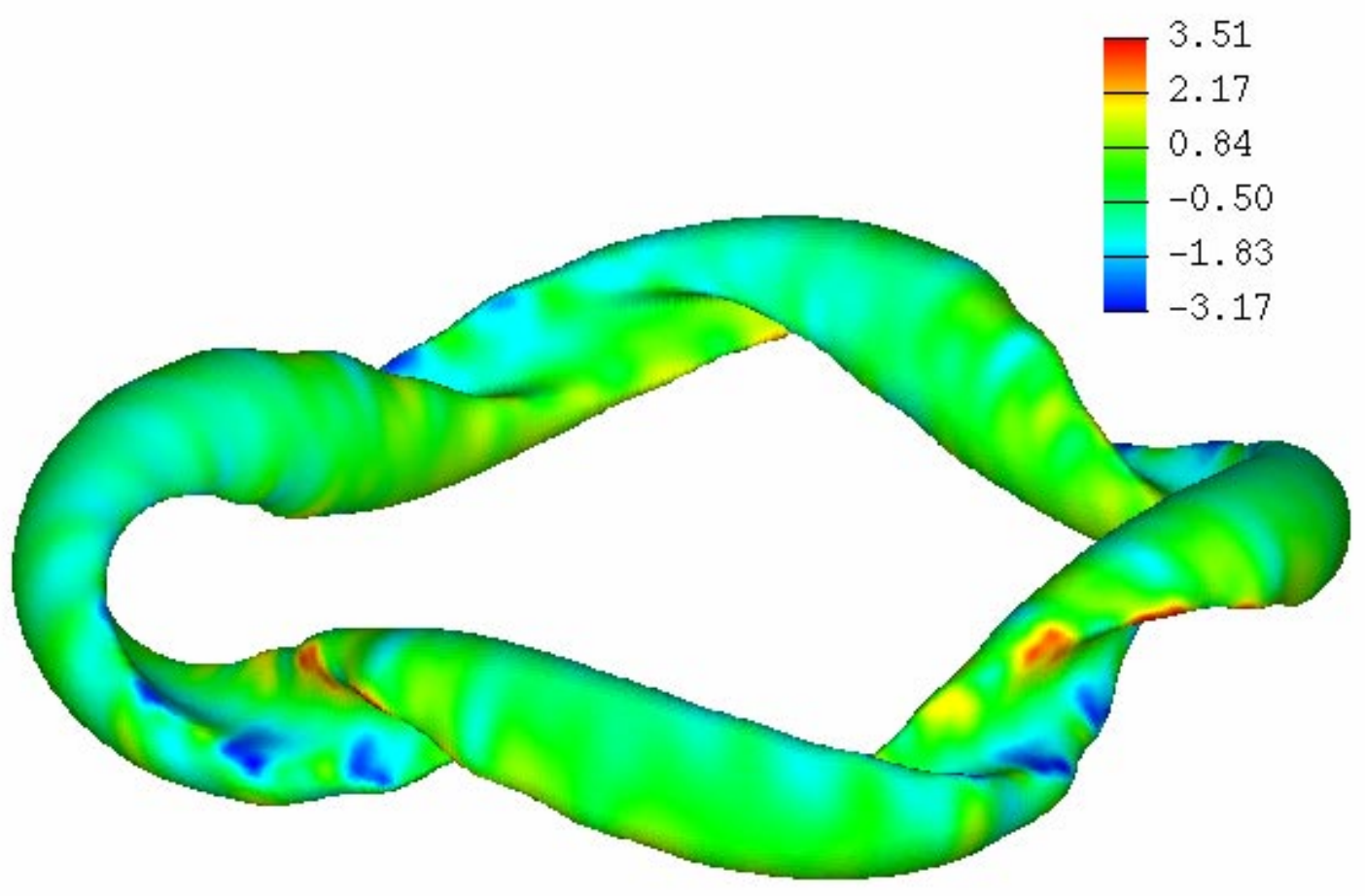

Figure 10: Geodesic magnetic curvature on a magnetic surface of stellarator TJ-II [22]. 


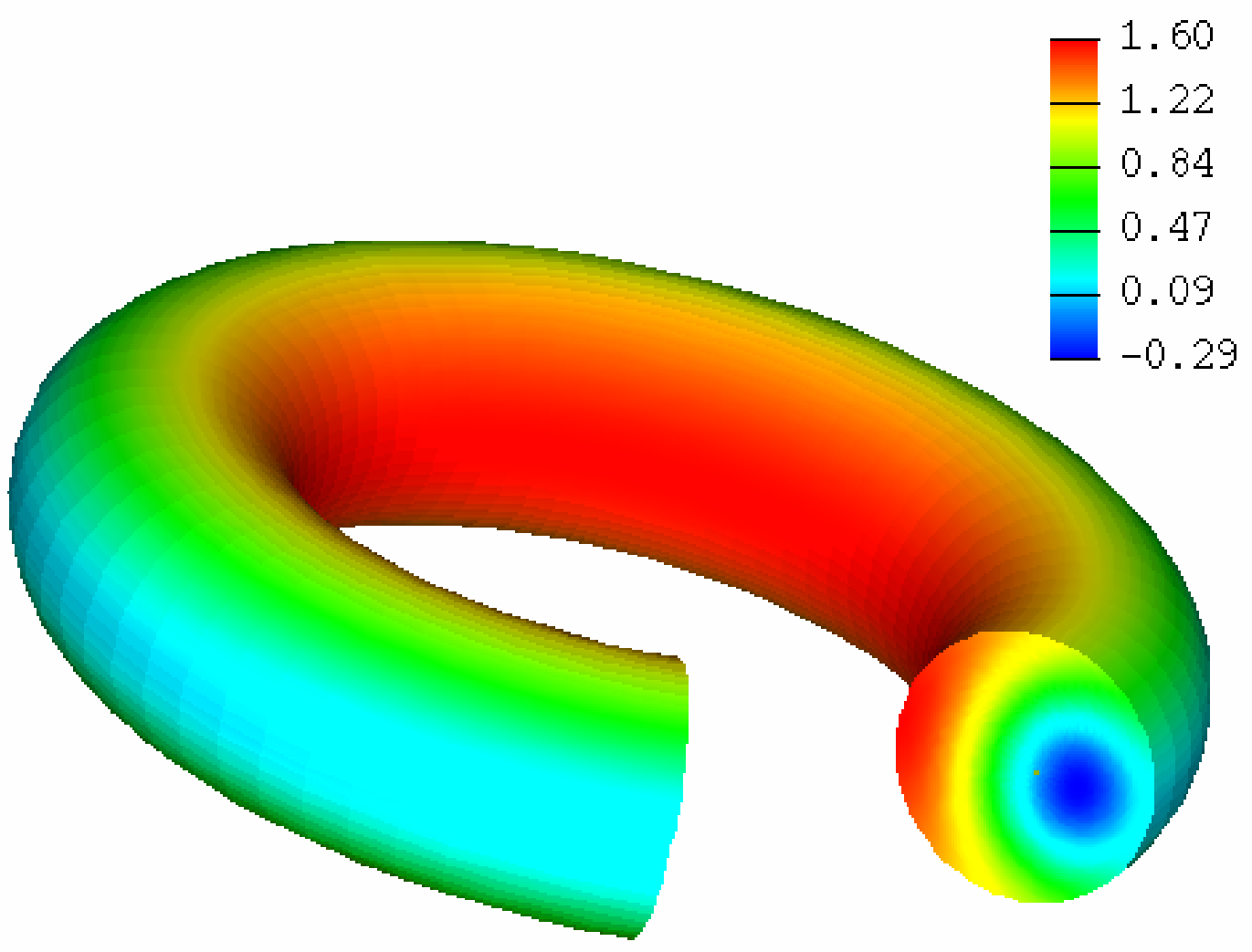

Figure 11: Local magnetic shear (LMS) for the standard tokamak. Blue indicates region with negative LMS. Positive values of the LMS are shown in red. 


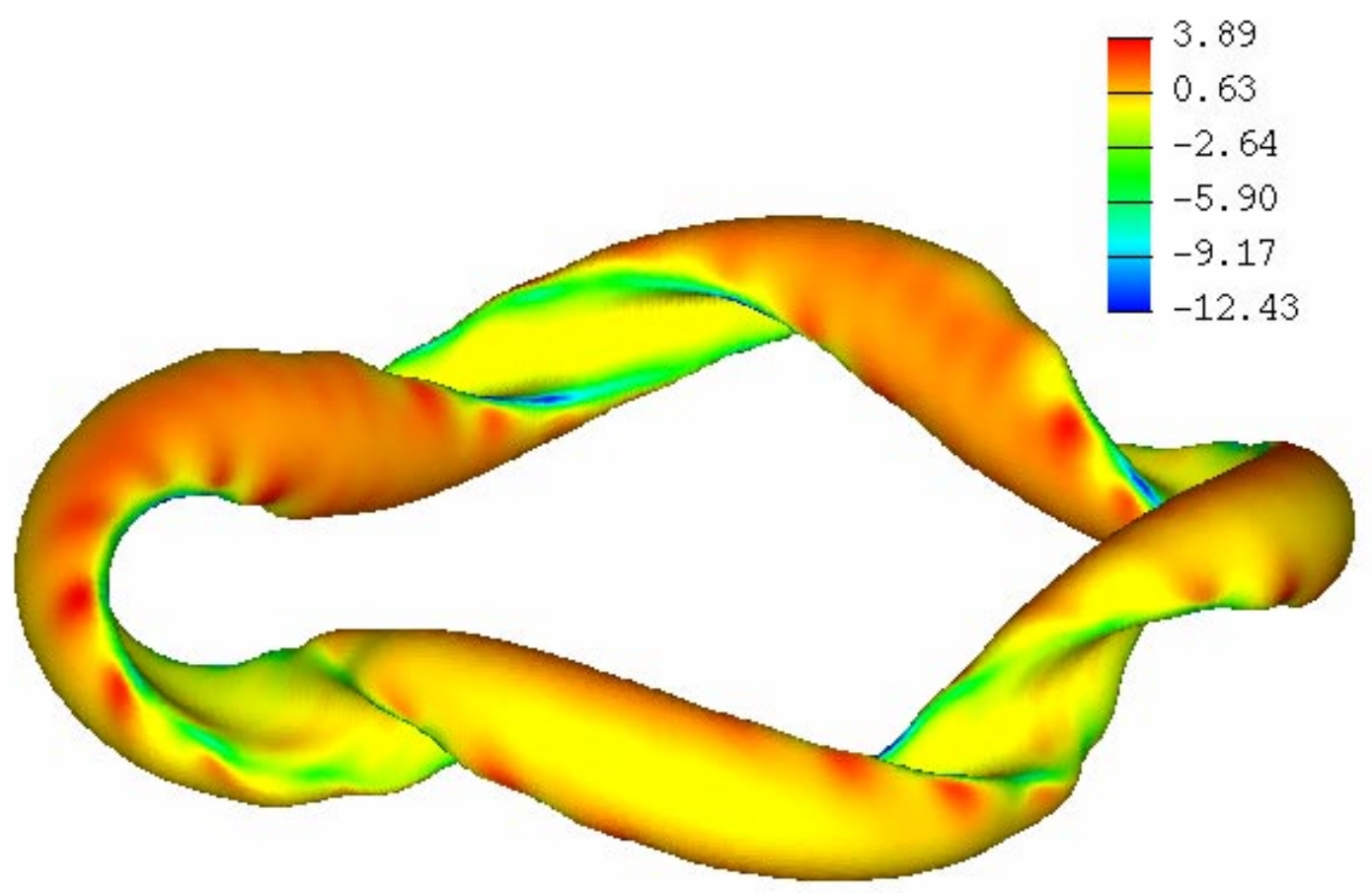

Figure 12: Local magnetic shear (LMS) on a magnetic surface of stellarator TJ-II [22]. 


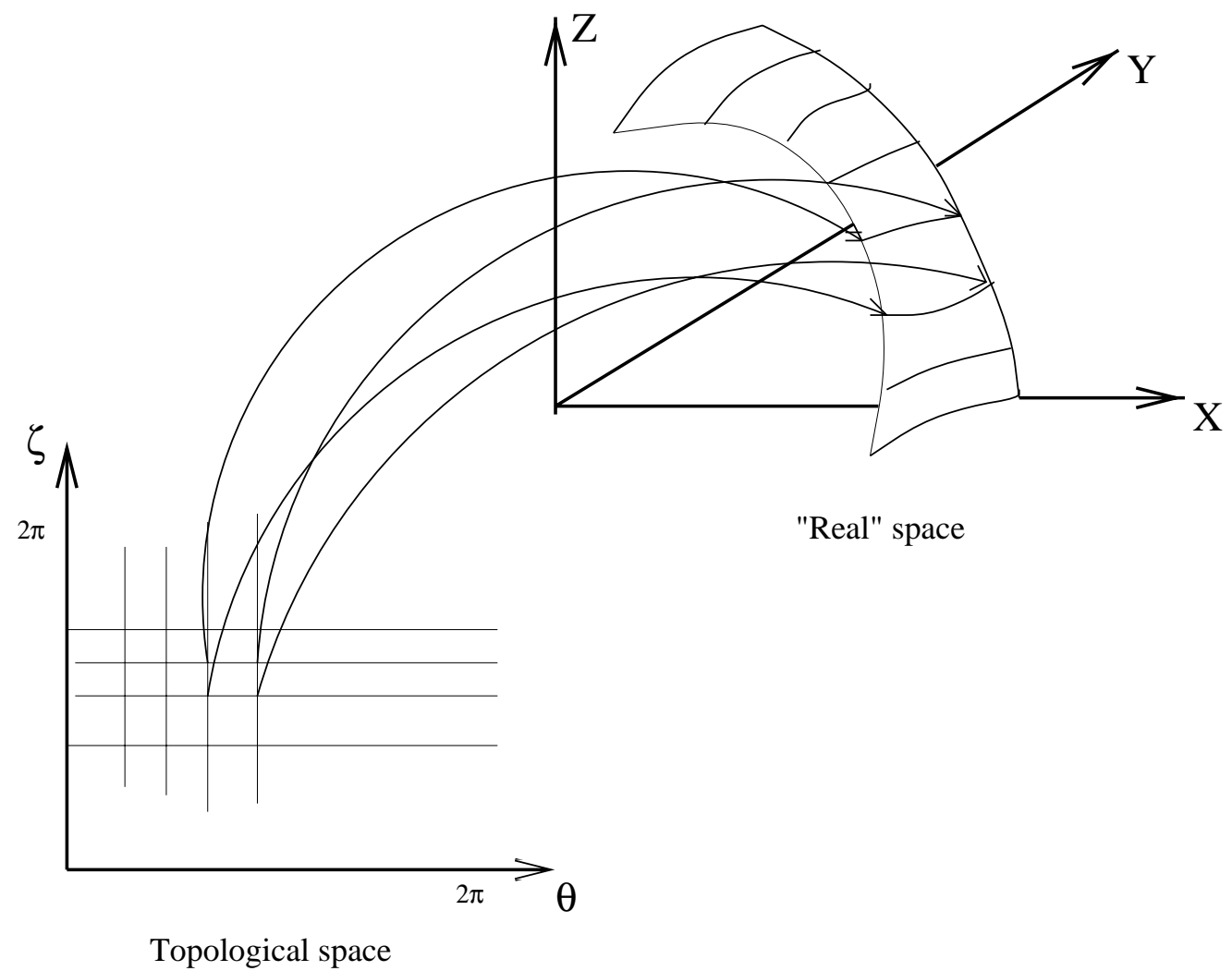

Figure 13: A magnetic surface is a square in topological space, but a fully 3-dimensional object in real space. 